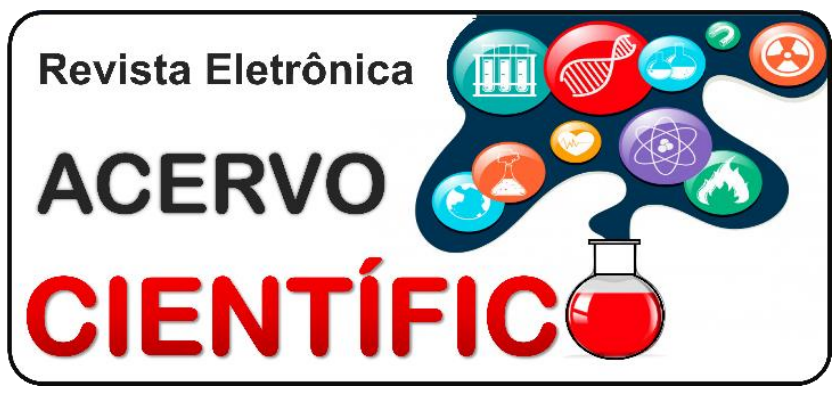

REVISÃO BIBLIOGRÁFICA

Recebido em: 7/2020

Aceito em: 8/2020

Publicado em: 11/2020

\title{
Complicações cardiovasculares e os impactos na qualidade de vida dos pacientes portadores da síndrome de Hutchinson-Gilford (HGPS)
}

\author{
Cardiovascular complications and the impacts on the quality of life of patients with \\ Hutching-Gilford syndrome (HGPS)
}
Complicaciones cardiovasculares e impactos en la calidad de vida de pacientes con síndrome de Hutchinson-Gilford (HGPS)

\begin{abstract}
Pedro Antonio Rodrigues Dias ${ }^{1 *}$, Maianna Viana Almeida Aguiar ${ }^{2}$, André Rhodes Neves ${ }^{2}$, Gabriela de Matos Reis ${ }^{1}$, Íris Roberta Cruz Andrade ${ }^{1}$, Isabella Pêgo Miranda Netto ${ }^{1}$, Leonardo José Grossi Andrade $^{1}$, Mariane de Matos Reis ${ }^{1}$, Mayra Heringer Motta Costa e Silva ${ }^{1}$, Sara Eduarda Oliveira da Cruz ${ }^{1}$.
\end{abstract}

\begin{abstract}
Resumo: Este artigo buscou revisar acerca de como as complicações cardiovasculares podem ser fatores agravantes na qualidade de vida dos pacientes portadores da Síndrome de Hutchinson-Gilford. A Síndrome de Hutchinson-Gilford (HGPS), conhecida como Progéria é uma patologia de envelhecimento precoce de origem genética autossômica dominante. Ocorre uma mutação no gene LMNA, resultando numa proteína anormal denominada progerina, que é responsável pelo aparecimento do fenótipo. A expectativa de vida para os portadores é de aproximadamente 14,6 anos, tendo como principal causa de morte as complicações cardiovasculares. Os principais resultados obtidos foram o surgimento de terapias gênicas fundamentais na tentativa de reduzir a transcrição de progerina. Por se tratar de uma patologia nova é importante um diagnóstico precoce por meio de testes genéticos para a alteração no gene LMNA. A terapia farmacológica empregada ainda está em estudo como, os Inibidores da farnesil transferase (FTIs), Pravastatina e ácido zoledrônico. Ademais, as associações dos três fármacos bloqueiam a evolução da patogenia do que apenas o uso isolado de cada um. Por fim, a importância da conscientização dos pais aos primeiros sinais de sintomas, além de suporte de uma equipe multidisciplinar e as mudanças nos hábitos de vida desses pacientes.
\end{abstract}

Palavras-chave: Progéria, Doenças cardiovasculares, Qualidade de vida.

Abstract: This article sought to review how cardiovascular complications can be aggravating factors in the
quality of life of patients with Hutchinson-Gilford Syndrome. Hutchinson-Gilford Syndrome (HGPS), known as
Progeria, is a pathology of premature aging of autosomal dominant genetic origin. A mutation occurs in the
LMNA gene, resulting in an abnormal protein called progerin, which is responsible for the appearance of the
phenotype. Life expectancy for patients is approximately 14.6 years, with cardiovascular complications as the
main cause of death. The main results obtained were the emergence of fundamental gene therapies in an

${ }_{1}^{1}$ Centro Universitário de Caratinga (UNEC), Caratinga - MG. *E-mail: pedrorr.dias99@gmail.com

2 União Educacional do Vale do Aço (UNIVAÇO), Ipatinga - MG. 
attempt to reduce the transcription of progerin. As it is a new pathology, an early diagnosis is important by means of genetic tests for the alteration in the LMNA gene. The pharmacological therapy used is still being studied, such as farnesyl transferase inhibitors (FTIs), Pravastatin and zoledronic acid. Furthermore, the associations of the three drugs block the pathogenesis evolution than just the isolated use of each one. Finally, the importance of parents' awareness of the first signs of symptoms, in addition to the support of a multidisciplinary team and changes in the lifestyle of these patients.

Keywords: Progeria, Cardiovascular diseases, Quality of Life.

Resumen: Este artículo buscaba revisar cómo las complicaciones cardiovasculares pueden ser factores agravantes en la calidad de vida de los pacientes con síndrome de Hutchinson-Gilford. El Síndrome de Hutchinson-Gilford (HGPS), conocido como Progeria, es una patología del envejecimiento prematuro de origen genético autosómico dominante. Se produce una mutación en el gen LMNA, lo que resulta en una proteína anormal llamada progerina, que es responsable de la aparición del fenotipo. La esperanza de vida de los pacientes es de aproximadamente 14,6 años, con complicaciones cardiovasculares como la principal causa de muerte. Los principales resultados obtenidos fueron la aparición de terapias genéticas fundamentales en un intento por reducir la transcripción de progerina. Como se trata de una nueva patología, un diagnóstico precoz es importante mediante pruebas genéticas para la alteración del gen LMNA. La terapia farmacológica empleada aún se está estudiando, como los inhibidores de farnesil transferasa (FTI), Pravastatina y ácido zoledrónico. Además, las asociaciones de los tres medicamentos bloquean la evolución de la patogénesis que solo el uso aislado de cada uno. Finalmente, la importancia de la conciencia de los padres sobre los primeros signos de síntomas, además del apoyo de un equipo multidisciplinario y los cambios en el estilo de vida de estos pacientes.

Palabras clave: Progeria, Enfermedades cardiovasculares, Calidad de vida.

\section{INTRODUÇÃO}

A Síndrome de Hutchinson-Gilford (HGPS), conhecida como Progéria é uma patologia de envelhecimento precoce de origem genética autossômica dominante (ROGGEN M, et al., 2017). É caracterizada por uma mutação do gene LMNA que codifica dois dos principais componentes da lâmina nuclear, a lâmina $\mathrm{A}$ e $\mathrm{C}$. Essa mutação é responsável pela ativação de um sítio de ligação alternativo, resultando numa proteína anormal denominada progerina, que é responsável pelo aparecimento do fenótipo. A expressão da progerina a nível celular leva a consequentes acúmulos na membrana nuclear, que está ligado a vários defeitos, como morfologia anormal das células, perda de heterocromatina, organização alterada das cromatinas, vias antioxidantes prejudicadas e deficiências na resposta a danos apresentados no DNA (RIVIERA M, et al., 2017; PIEKAZOWICZ K, et al., 2019).

A HGPS afeta 1 a cada 4-8 milhões de neonatos e a expectativa de vida para os portadores é de aproximadamente 14,6 anos, tendo como principal causa de morte as complicações cardiovasculares. As crianças com HGPS parecem saudáveis ao nascimento, mas começam a exibir características clínicas para diagnóstico já no primeiro ano de vida, como retardo do crescimento, alopecia, pele envelhecida, rigidez articular e densidade óssea reduzida (ROGGEN M, et al., 2017; VIDAK S e FOISNER R, 2016). Curiosamente a patologia da HGPS não afeta o sistema nervoso central (SNC), assim paciente acometidos detém a função cerebral preservada, sem comprometimento cognitivo ou alteração na memória que não seja normal do envelhecimento (GONZALO S e COLL-BONFILL N, 2019).

Dentre as diversas alterações clínicas, os pacientes portadores da HGPS desenvolvem alterações cardiovascular isoladas, sendo as principais complicações a aterosclerose e a insuficiência cardíaca. Além disso, os defeitos relacionados à idade se manifestam na condução elétrica do coração com intervalo QT prolongado, depressão e elevação do segmento ST e ondas T negativas ou bifásicas na eletrocardiografia (ECG). Dessa forma, tais manifestações interferem diretamente na qualidade de vida desses pacientes e 
ocasionam precocemente a morte no início da adolescência devido a acidente vascular cerebral ou infarto no miocárdio (MESSNER M, et al., 2018; HAMCZYK MR e ANDRÉS V, 2019).

Até o momento não existem terapias que possam interferir nos mecanismos moleculares e impedir a progressão da síndrome, logo, o manejo é realizado por uma equipe multidisciplinar voltado para o alívio dos sintomas, prática de atividades físicas e monitoramento de órgãos vitais (GONZALO S e COLL-BONFILL N, 2019; ARANIBAR L, et al., 2013).

Para tanto, define-se como objetivo do presente trabalho fazer uma revisão acerca de como as complicações cardiovasculares podem ser fatores agravantes na qualidade de vida dos pacientes portadores de Síndrome de Hutchinson-Gilford.

\section{REVISÃO BIBLIOGRÁFICA}

\section{Fisiopatologia}

A HGPS é causada por uma mutação no gene LMNA presente no cromossomo 1, onde ocorre uma substituição do nucleotídeo citosina por timina durante o processo de transcrição. Na tradução, com o RNAm alterado, devido a uma deleção de 50 aminoácidos próximo a extremidade C-terminal forma a proteína Progerina (GORDON LB, et al., 2019).

Normalmente o gene LMNA codifica a lâmina do tipo A e por splicing alternativo origina as isoformas A e C. Essa lâmina é um componente da membrana nuclear responsável por sua rigidez, além de possuir um papel mecânico-químico tem função de organizar a cromatina, os genes de regulação de reparo de ácido desoxirribonucleico (DNA) e a sinalização (VIDAK S e FOISNER R, 2016; STRANDGREN C, et al., 2017). Outras variantes do gene LMNA não levam a produção da proteína progerina, mas sim de lâmina $A$ anormais em sua estrutura e função, resultando em doenças celulares e de organismos com fenótipo característico dos pacientes com progeroides (GORDON LB, et al., 2019).

A progerina é uma proteína que insere na membrana nuclear interna, onde se acumula causando danos celulares tais como: encurtamento de telômeros, estresse oxidativo, dano ao DNA, perda de heterocromatina e também a prematuridade da senescência celular (GONZALO S e COLL-BONFILL N, 2019).

Os pacientes com progéria possuem telômeros muito curtos levando ao envelhecimento da pele, das células musculares, cardiovasculares e do sistema nervoso central. O comprimento do telômero diminui em cada replicação, chegando a uma determinada posição onde não ocorre mais a divisão, causando a senescência celular (AHMED MS, et al., 2017). Os fibroblastos de pacientes com HGPS provoca aumento de espécies reativas de oxigênio (ERO), o seu excesso pode levar ao estresse oxidativo, induzindo a senescência e/ ou apoptose celular (KANG HT, et al., 2017).

\section{Manifestações clínicas}

As crianças com HGPS começam a apresentar várias manifestações clínicas decorrentes da patologia no primeiro ano de vida, como retardamento no crescimento, alopecia, diminuição de gordura subcutânea, olhos proeminentes, aparência de pele envelhecida, rigidez articular e densidade óssea reduzida. Quando se tornam mais velhas, sofrem de osteoporose, aterosclerose e complicações cardiovasculares (VIDAK S e FOISNER R, 2016).

Analisando especificamente as manifestações clínicas cardiovasculares, já foram descritas 16 alterações causadas pela HGPS: insuficiência cardíaca, disfunção diastólica do ventrículo esquerdo, hipertrofia ventricular, fibrose cardíaca, disfunção da válvula cardíaca, aumento da atividade simpática, diminuição da sensibilidade barorreflexa, espessamento da camada íntima e média, enrijecimento vascular, fibrose vascular, disfunção endotelial, aterosclerose, calcificação vascular, hipertensão, elevação da quantidade de plaquetas e prolongamento do tempo de protrombina (AHMED MS, et al., 2017).

A insuficiência cardíaca, como uma das alterações funcionais estruturais, manifesta-se a partir da dispneia e da fadiga, com capacidade limitada de exercitar-se e retenção líquida, podendo resultar em um edema 
pulmonar e de extremidades. Juntamente com ela, ocorre a disfunção diastólica do ventrículo esquerdo, que é quando o mesmo perde sua capacidade de relaxar normalmente, devido ao enrijecimento do músculo cardíaco pós fibrose, e a hipertrofia ventricular esquerda, onde há um aumento na espessura da parede do ventrículo esquerdo, muitas das vezes causada por hipertensão, obesidade, doenças valvares e infarto do miocárdio (HAMCZYK MR, et al., 2017).

Ainda, o aumento de colágeno na matriz extracelular (ECM) resulta em fibrose cardíaca e é um dos principais fatores para a insuficiência cardíaca, já que reduz a habilidade do miocárdio conduzir os impulsos elétricos, relaxar e contrair corretamente, e difundir oxigênio, aumentando casos de arritmia e outras disfunções valvares. Em relação à disfunção da válvula cardíaca, acontece devido ao aumento de grossura e de rigidez das válvulas mitral e aórtica após calcificação e degeneração do tecido conjuntivo. Sobre a arritmia, ela acontece devido ao coração envelhecido possuir capacidade reduzida para aumentar a frequência cardíaca, diminuindo o output cardíaco. A desregulação cardiovascular é responsável pelo aumento da atividade simpática, levando em conta o aumento também dos níveis plasmáticos de catecolaminas e a diminuição da sensibilidade beta-adrenérgica e da sensibilidade barorreflexa (HAMCZYK MR, et al., 2017).

Sobre as alterações vasculares em pacientes com HGPS, cita-se a hipertensão, onde o aumento da atividade simpática aumenta a pressão do sangue. Além dela, tem-se a rigidez vascular, a disfunção endotelial. Tal rigidez arterial pode levar à fibrose e insuficiência cardíaca, além de iniciar a progressão da hipertensão e da aterosclerose, após promover a disfunção celular na parede do vaso. A disfunção endotelial altera a função da célula homeostática no endotélio, incluindo o controle do tônus e da permeabilidade vascular, além de inflamação na membrana (HAMCZYK MR, et al., 2017; DEL CAMPO L, et al., 2018).

Cerca de 30\% dos pacientes apresentam apenas uma elevação da pressão sanguínea sistólica e diastólica comparando com crianças saudáveis da mesma idade. Além disso, apresentam níveis elevados de plaquetas e prolongado tempo de protrombina (HAMCZYK MR, et al., 2017), além de haver a geração de uma fibrose mais elevada e densa rica em colágeno e em proteoglicanos. A maioria das lesões nos pacientes com a síndrome apresenta grandes regiões de calcificação (lesões fibro-calcificadas) e a progerina está bem representada em todas as camadas da vasculatura coronariana (OLIVE M, et al., 2010).

Ademais, também apresentam insuficiência ventricular - que demonstra a degeneração generalizada do tecido, além do inevitável bloqueio severo dos vasos - e aterosclerose, que é geralmente associada de forma direta ao envelhecimento causado pela doença, sendo uma consequência da cicatrização da parede arterial pós lesão (DEL CAMPO L, et al., 2018). Essas lesões arteriais tendem a possuir núcleos ateromatosos menores devido à falta de hipercolesterolemia e dislipidemia nesses pacientes e o acúmulo de progerina nas paredes arteriais e nas placas íntimas pode ser um fator importante subjacente ao desenvolvimento dessas lesões vasculares prematuras e progressivas (OLIVE M, et al., 2010).

É importante ressaltar que a patologia do HGPS não interfere no sistema nervoso central (SNC), isso porque, o gene LMNA no cérebro produzem primeiro o Lâmina $C$ e não o Lâmina $A$ ou a progerina. Isto ocorre devido à expressão de um microRNA, miR-9, que tem como alvo específico a prelamin $\mathrm{A} /$ transcritos de progerina no cérebro. Pacientes que possuem a progeria apresentam a função cerebral normal, sem alterações cognitivas (GONZALO S e COLL-BONFILL N, 2019). Em suma, as crianças com essa síndrome têm a capacidade de se desenvolver bem em ambientes sociais, sendo preconizado uma escolaridade apropriada à idade (GORDON LB, et al., 2019).

\section{Diagnóstico}

A detecção precoce é difícil, pois os bebês com HGPS parecem saudáveis ao nascerem. O diagnóstico geralmente é dado entre 12 a 18 meses, até o segundo ano de vida, fase em que começam a ter as alterações nas características clínicas e anormalidades da doença como falha no crescimento, dentição anormal, ponte nasal estreita, ponta do nariz pontiaguda, mandíbula recuada, alopecia parcial ou completa, veias proeminentes do couro cabeludo, pele esclerodérmica, fraqueza muscular, alterações no perfil lipídico sérico com perda de gordura subcutânea, resistência à insulina, membros finos, osteoporose, osteólise das falanges terminais, coxa valga entre outras manifestações (SAXENA S e KUMAR S, 2020; AHMED MS, et al., 2017). 
No passado só era possível diagnosticar o HGPS usando informações clínicas, como uma aparência geral e raios- $X$, levando a frequentes diagnósticos incorretos. Portanto, hoje já se tem teste genético para a mutação no gene LMNA em que confirma a presença da progéria. Após diversos estudos cientistas descobriram que a síndrome é causada por uma alteração de uma letra que compõem o genoma humano (DNA). Essa mudança pode ser vista através do teste usando o sequenciamento genético, no qual o gene é decodificado e sua sequência é determinada letra por letra (ULLRICH NJ e GORDON LB, 2015). Sendo assim, isso pode garantir diagnósticos mais precisos e precoces com menos erros, além de intervenção médica antecipada para garantir uma melhoria na qualidade de vida das crianças acometidas.

\section{Tratamento}

Mediante a fisiopatologia da doença, não existe ainda um tratamento eficaz ou cura para a HGPS. Os recursos terapêuticos são acompanhamentos multiprofissional e medidas paliativas, em que procuram minimizar os sintomas com medidas como acompanhamento nutricional com uma dieta rica em calorias junto a polivitamínico para nutrição adequada e redução da perda de peso. Também é recomendado a hidratação oral adequada, visto que os vasos sanguíneos mais rígidos podem ter menos tolerância à desidratação. São realizadas avaliações cardíacas seriadas, como eletrocardiograma (ECG) para detecção e prevenção de aterosclerose da artéria coronária. As crianças também podem ser tratadas com doses menores de aspirina para diminuir as chances de coágulos, ataques cardíacos e derrames, e caso tenham angina podem ser tratadas com nitroglicerina (SAXENA S e KUMAR S 2020; ULLRICH NJ e GORDON LB, 2015).

Como método de minimizar as repercussões clínicas, algumas pesquisas apontam para uma terapia gênica, em que se bloqueia a expressão da progerina juntamente com a expressão da Lâmina $A$ e isso pode trazer resultados benéficos a esses pacientes. Uma das estratégias foi apresentada em um estudo, no qual introduziram oligonucleotídeos em fibroblastos de HGPS ou linfócitos B fazendo com que ocorresse uma diminuição da transcrição de progerina (PIEKAROWICZ K, et al., 2019).

Vários outros modelos farmacológicos estão em processo de pesquisa por serem um potencial terapêutico contra a síndrome de Hutchinson-Gilford. Segundo comprova diversos estudos, os Inibidores da farnesil transferase (FTIs) que foram inicialmente utilizados para o tratamento de câncer, foram os que demonstraram maior eficácia contra o fenótipo da doença, sendo capaz de restaurar a morfologia nuclear das células afetadas pela HGPS e melhorar a capacidade de auto renovação em células-tronco que expressam progéria (SAXENA S e KUMAR S, 2020).

Em 2010 foi realizado um teste científico, por meio de incentivo da associação Progéria Research Foundation, Institutos Nacionais de Saúde, Hospital Infantil Boston e Instituto de Câncer Dana-Farber com intuito de avaliar os três medicamentos que obtiveram alguma resposta positiva, que foram o Pravastatina, Lonafarnib e ácido zoledrônico, com objetivo final de achar a cura para a Síndrome de Hutchinson-Gilford. Contudo, o Lonafarnib, um FTI (inibidor da farnesil transferase) está no estágio 2 da pesquisa sendo testado em crianças com a doença, foi o que se mostrou mais vantajoso sendo capaz de curar várias condições cardiovasculares relacionadas à síndrome, apresentou também eficiente melhora nos distúrbios ósseos e ajudou a ganhar adipócitos nos pacientes afetados (SHARMA V e SHUKLA R, 2019).

Outro medicamento importante é a Pravastatina membro da classe de drogas das estatinas, geralmente é usado para baixar o colesterol e prevenir doenças cardiovasculares. Ela foi considerada útil na síndrome pois verificou melhora nos problemas cardiovasculares e diminuição dos níveis de colesterol das crianças. $O$ terceiro medicamento que também foi submetido ao teste científico é o ácido zoledrônico ele é um bisfosfonato, usado como medicamento ósseo para melhorar a osteoporose e prevenir fraturas esqueléticas. Foi útil segundo os estudos para evitar feridas ou fissuras ósseas, além de melhorar a osteoporose dos pacientes (SHARMA V e SHUKLA R, 2019).

A coxa valga e a luxação de quadril são outras comorbidades muito comuns nas crianças com HGPS, por isso é recomendado incentivo para que sejam fisicamente ativas dentro de suas limitações além de fisioterapia, órtese corporal e procedimentos cirúrgicos caso necessário, a terapia física e ocupacional é sugerida para manter a amplitude do movimento nas articulações. Portanto, é necessário o acompanhamento 
de fisioterapeutas e ortopedistas com as crianças por estarem mais susceptíveis a fraturas. Além disso, é sugerida a extração dos dentes primários no intuito de evitar o apinhamento dentário a suplementação com fluoreto também é recomendada para reduzir problemas dentários. Essas e outras medidas que podem ser realizadas ajudam a melhorar a qualidade de vida e por consequência diminuem as complicações clínicas, porém não são capazes de levar a cura (ULLRICH NJ e GORDON LB, 2015).

\section{Prognóstico}

Os pacientes que apresentam HGPS possuem uma baixa longevidade e morrem em sua maioria na adolescência. Cerca de apenas 150 casos estão descritos na literatura, predominando no sexo masculino com relação de 1,5:1 (LIMA LL, et al., 2011). Não é uma doença restrita a uma etnia, localização demográfica ou a qualquer estatuto socioeconômico (HENNEKAM RC, 2006).

Apesar dos avanços relacionados à cirurgia cardiovascular, a baixa taxa de sobrevida mantém-se devido a alta capacidade de produção das placas ateromatosas, e dessa maneira, o principal fator de mortalidade são as doenças cardiovasculares (75\%) como o infarto agudo do miocárdio o acidente vascular cerebral (AVC) (MERIDETH MA, et al., 2008).

Não é frequente observar outros sintomas degenerativos como: fragilidade imunitária, déficit neurológico, cataratas e diabetes mellitus tipo II. Contudo, em casos de ceratopatia de exposição os lubrificantes oculares são eficazes e os aparelhos auditivos podem ser usados quando clinicamente necessários. Além disso, notase que os portadores acabam por ficar com algum cabelo residual ou sem cabelo nenhum, e mesmo acontece com sobrancelhas e pelos do corpo (MERIDETH MA, et al., 2008; GORDON LB, et al., 2019).

\section{CONSIDERAÇÕES FINAIS}

Levando em consideração o que foi apresentado, os pacientes portadores da HGPS produzem progerina em abundância, fator este, responsável pelo processo de envelhecimento precoce e que vão ser responsáveis pelo surgimento das complicações cardiovasculares e consequentemente a diminuição na expectativa de vida das crianças acometidas. Apesar de ser uma patologia nova e extremamente rara, verifica-se a importância do diagnóstico precoce por meio de testes genéticos que buscam encontrar alterações no gene LMNA, e dessa maneira instruir os cuidados necessários para aliviar os sintomas e retardar o surgimento dos novos. Associado a isso, é de suma importância a conscientização dos pais para se atentarem aos primeiros sintomas, visto que normalmente aparecem no primeiro ano de vida. Em virtude dos fatos mencionados, o aumento do número de publicações sobre a patologia, bem como a assistência dos acometidos por uma equipe multidisciplinar e mudanças nos hábitos diários podem ser fundamentais na manutenção da qualidade de vida desses pacientes que tanto sofrem por essa patologia.

\section{REFERÊNCIAS}

1. AHMED MS, et al. Hutchinson-Gilford Progeria Syndrome: A Premature Aging Disease. Mol Neurobiol. 2017.

2. ARANIBAR DL, et al. Enfermedades hereditarias de la dermis: revisión de la literatura / Hereditary diseases of the dermis: literature review. Revista Chilena de Dermatologia; 29(1): 38-51, 2013.

3. DEL CAMPO L, et al. Mecanismos de envejecimiento vascular: ¿Qué podemos aprender del síndrome de progeria de Hutchinson-Gilford? Sociedad Espanola de Arteriosclerosis vol. 30,3 (2018): 120-132.

4. GONZALO S, COLL-BONFILL N. Genomic instability and innate immune responses to self-DNA in progeria. Geroscience. 2019;41(3):255-266.

5. GORDON LB, et al. Hutchinson-Gilford Progeria Syndrome, National Library of Medicine, 2019.

6. HAMCZYK MR, et al. Aging in the Cardiovascular System: Lessons from Hutchinson-Gilford Progeria Syndrome. Annual Review of Physiology. 2017.

7. HAMCZYK MR, ANDRÉS V. Vascular smooth muscle cell loss underpins the accelerated atherosclerosis in Hutchinson-Gilford progeria syndrome. Nucleus. 2019; 10:1, 28-34

8. HENNEKAM RC. Hutchinson-Gilford progeria syndrome: Review of the phenotype. Am J Med Genet Part A, 140A, 2006; 2603-2624. 
9. KANG HT, et al. Chemical screening identifies ROCK as a target for recovering mitochondrial function in HutchinsonGilford progeria syndrome. Aging Cell, 2017; 16, pp541-550

10. LIMA, LL, et al. Você conhece esta síndrome? An. Bras. Dermatol., 2011, 86, 165-166

11. MERIDETH MA, et al. Phenotype and Course of Hutchinson-Gilford Progeria Syndrome. The New England Journal of Medicine, 2008; 358:592-604.

12. MESSNER M, et al. "Upregulation of the aging related LMNA splice variant progerin in dilated cardiomyopathy." PLoS One 13.4 (2018): e0196739.

13. OLIVE M, et al. Cardiovascular Pathology in Hutchinson-Gilford Progeria: Correlation with the Vascular Pathology of Aging. Arterioscler Thromb Vasc Biol. Nov 2010.

14. PIEKAZOWICZ K, et al. Hutchinson-Gilford Progeria Syndrome-Current Status and Prospects for Gene Therapy Treatment. Cells. 2019;8(2):88

15. PRAKASH A et al. Cardiac Abnormalities in Patients With Hutchinson-Gilford Progeria Syndrome, JAMA Cardiology, 2018

16. RIVERA M, et al. DNA replication timing alterations identify common markers between distinct progeroid diseases." Proceedings of the National Academy of Sciences; 2017; 114.51

17. ROGGEN M, et al. Coronary artery stenting in a patient with progeria. Catheterization and Cardiovascular Interventions; 2017; E38-E40.

18. SAXENA S, KUMAR S. Pharmacotherapy to gene editing: potential therapeutic approaches for Hutchinson-Gilford progeria syndrome. Geroscience. 2020;42(2):467-494.

19. SHARMA V, SHUKLA R. Progeria: A Rare Genetic Syndrome. Indian J Clin Biochem. 2020;35(1):3-7

20. STRANDGREN $C$, et al. Emerging candidate treatment strategies for Hutchinson-Gilford progeria syndrome. Biochemical Society Transactions, 2017; 45, 1279-1293.

21. ULLRICH NJ, GORDON LB. Hutchinson-Gilford progeria syndrome. Handbook of Clinical Neurology, 2015; 249-264

22. VIDAK S, FOISNER R. Molecular insights into the premature aging disease progeria. Histochem Cell Biol, 2016; 145:401-417.

23. WORMAN HJ, MICHAELIS S. Permanently Farnesylated Prelamin A, Progeria, and Atherosclerosis, Circulation, 2018 